\title{
Distribución y abundancia de crustáceos en humedales de Tabasco, México
}

\author{
Abundance and distribution of crustaceans in wetlands of Tabasco
}

\author{
Everardo Barba*, Juan Juárez-Flores y Feliciana Estrada-Loreto \\ Departamento de Aprovechamiento y Manejo de Recursos Acuáticos (DAMRA), Área de Sistemas de Producción Alternativos, El Colegio de la \\ Frontera Sur, Unidad Villahermosa. Km. 15.5 carretera Villahermosa-Reforma s/n, R/a Guineo Segunda Sección, 86280 Villahermosa, Tabasco, \\ México. \\ *Correspondencia: ebarba@ecosurmx
}

\begin{abstract}
Resumen. El estado de Tabasco presenta una compleja y alta diversidad de humedales que incluye tipos palustres $(20.72 \%)$, costeros $(3.93 \%)$, lacustres $(1.74 \%)$ y ribereños $(1.37 \%)$, los cuales cubren el $27 \%$ del territorio estatal. La diversidad faunística en estos ambientes comprende invertebrados y vertebrados, donde la mayor parte de los registros son de especies de interés comercial. Se presenta el inventario de crustáceos recolectados desde 2003 hasta la fecha en las subregiones Sierra, Ríos y Pantanos. La recolección fue diurna, en 14 localidades (ambientes lóticos y lénticos), mediante nucleador y draga para la infauna, y arrastres con diferentes artes para la epifauna. Se obtuvieron 2370 organismos que pertenecen a 4 órdenes, 15 familias, 17 géneros y 17 especies. Las especies más abundantes fueron Discapseudes holthuisi Bacescu y Gutu, 1975 (62\%), Macrobrachium acanthurus (Wiegmann, 1836) (12\%), Leptochirus sp. Zaddach, 1844 (8\%) y Palaemonetes vulgaris (Say, 1818) (7\%), sumando el 89\% del total. El 67\% de la abundancia correspondió a la infauna y el 33\% a la epifauna. El 81\% de los crustáceos se capturaron en sistemas lénticos y el resto en sistemas lóticos. Este trabajo contribuye con nuevos registros de crustáceos en humedales de Tabasco.
\end{abstract}

Palabras clave: crustáceos, humedales dulceacuícolas, Tabasco.

\begin{abstract}
The state of Tabasco is characterised by complex and diverse wetlands of different types: palustrine (20.72\%), coastal (3.93\%), lacustrine (1.74\%) and riverine (1.37\%), covering $27 \%$ of its surface. Faunal diversity of these wetlands includes invertebrates and vertebrates, most of the available records come from species with commercial importance. The list of crustaceans collected from 2003 to date in the subregions Sierra, Ríos and Pantanos, is presented. The sampling at 14 localities was diurnal (lotic and lentic environments), using cores and dredges for the infauna and different nets for epifauna. A total of 2370 organisms belonging to 4 orders, 15 families, 17 genera and 17 species, was obtained. The most abundant species were: Discapseudes holthuisi Bacescu y Gutu, 1975 (62\%), Macrobrachium acanthurus (Wiegmann, 1836) (12\%), Leptochirus sp. Zaddach, 1844 (8\%) and Palaemonetes vulgaris (Say, 1818) (7\%), accounting for 89\% of the total abundance. Infaunal abundance represented $67 \%$ and epifaunal 33\%. Eighty-one percent of the organisms were collected in lentic environements and $19 \%$ in lotic systems. The present study contributes with new records of crustaceans for the wetlands of Tabasco.
\end{abstract}

Key words: crustaceans, freshwater wetlands, Tabasco.

\section{Introducción}

El estado de Tabasco ocupa el $1.3 \%$ del territorio nacional y el $27.7 \%$ de su superficie está cubierta por humedales. Estos ecosistemas acuáticos proporcionan hábitat para una gran diversidad de especies, tanto de importancia ecológica como económica. La diversidad faunística en Tabasco cuenta con un total de 896 especies acuáticas y 227 terrestres, entre los registros de especies acuáticas destacan los artrópodos acuáticos, moluscos

Recibido: 12 noviembre 2009; aceptado: 26 abril 2010 y peces; los mejor representados son los moluscos con 164 especies (Sánchez y Barba, 2005), y los crustáceos decápodos con un total de 72 especies (Álvarez et al., 2005).

La proporción de humedales está dominada por sistemas palustres en el $21 \%$, los costeros ocupan un $4 \%$, mientras que los lacustres y ribereños el 1.7 y $1.28 \%$, respectivamente (Barba et al., 2006). En nuestro país, los humedales constituyen unidades ambientales donde concurren múltiples intereses económicos, dada la variedad de recursos que en ellos se encuentran. Estos sistemas están siendo destruidos o modificados sustancialmente, entre 
otros factores, por operaciones de drenado y relleno para ganar terreno para la agricultura, construcción de presas, desarrollos urbanos, aporte de sedimentos por erosión, descarga de contaminantes tóxicos y eutrofización, como resultado de malas prácticas agrícolas. En consecuencia ha disminuido su valor y función ecológica natural, al reducirse el nivel de captación de agua y la calidad del hábitat para la fauna silvestre (de la Lanza y GarcíaCalderón, 1995; Sánchez y Barba, 2005).

La alta diversidad de recursos acuáticos distribuidos en las subregiones de Tabasco, las condiciones ecológicas y el grado de conservación de éstos influyen sobre la distribución y abundancia de los organismos acuáticos; por otra parte, se encuentran los factores dependientes del régimen de precipitación, el grado y la amplitud de la inundación (Ramírez y Roldán, 1989; Araúz et al., 2000; Rincón, 2003; Hurtado et al., 2005). No obstante la importancia de los sistemas acuáticos en general, los ambientes estuarinos han recibido mayor atención de investigación por ser zonas de reproducción, crianza y refugio de una gran cantidad de organismos acuáticos estuarino-marinos de importancia ecológica y comercial (Yáñez-Arancibia, 1986). Lo anterior se constata por el mayor número de registros para la zona costera; la subregión de la Chontalpa cuenta con el $68 \%$ de las especies registradas y la subregión Ríos con el 24\% (Sánchez y Barba, 2005).

Entre la fauna representativa de los humedales se encuentran los crustáceos, los cuales desempeñan un papel importante en la transferencia energética hacia niveles tróficos superiores (Barba et al., 2000); algunos pueden emplearse como indicadores de la calidad del agua (Álvarez et al., 1996; de la Lanza et al., 2000) y otros, predominantemente marinos (89\%), se capturan con fines de consumo. Debido a sus variadas adaptaciones, entre las que destacan la respiración aérea (Díaz y Rodríguez, 1977) y la capacidad de osmorregulación de iones minerales (Mantel y Farmer, 1983), estos organismos han invadido tanto ambientes dulceacuícolas como terrestres representando respectivamente el $10 \%$ y $1 \%$ de la diversidad (Álvarez et al., 1996).

Aún reconociendo la importancia de los humedales como hábitat, el estado de conocimiento de la biodiversidad de estos ambientes permanece incompleto, lo cual se refleja en los listados faunísticos, en particular en cuanto al número de especies de macroinvertebrados en general. Esto se atribuye a una subestimación en las áreas de captura y a un bajo esfuerzo de recolección de los diversos grupos que componen esta fauna y también por su taxonomía compleja (Sánchez y Barba, 2005). Este trabajo contribuye con nuevos registros de distribución y abundancia de crustáceos en los humedales de Tabasco.

\section{Materiales y métodos}

Área de estudio. El estado de Tabasco se localiza en el sureste de México, con una superficie de $24661 \mathrm{~km}^{2}$, cubre desde la planicie costera del golfo de México, hasta las montañas del norte de Chiapas, se ubica entre $\operatorname{los} 17^{\circ} 15^{\prime}$ y $18^{\circ} 39^{\prime} \mathrm{N}$ y los $91^{\circ} 00^{\prime}$ y $94^{\circ} 07^{\prime} \mathrm{O}$. Tabasco representa el $1.3 \%$ del territorio nacional, se divide en 5 subregiones: Chontalpa, Ríos, Sierra, Centro y Pantanos. Predominan los climas cálido-húmedo con lluvias todo el año, cálido-húmedo con abundantes lluvias de monzón y cálido-húmedo con lluvias en verano, con una temperatura media anual de $26^{\circ} \mathrm{C}$ y máxima de $42^{\circ} \mathrm{C}$ (INEGI, 2008). Las localidades estudiadas abarcaron la heterogeneidad de los humedales dulceacuícolas en las subregiones de Tabasco (Fig. 1; Cuadro 1).

Caracterización ambiental. Se cuantificaron las condiciones ambientales en las localidades de muestreo. Las variables físico químicas evaluadas in situ fueron: $\mathrm{pH}$, temperatura, concentración de oxígeno disuelto (OD), y las evaluadas ex situ: nitratos y amonio en agua y $\mathrm{pH}$ en sedimento (Cuadro 2), mediante sondas multiparamétricas de la marca HANNA, modelos HI-9818 y HI-98185, respectivamente. Las muestras de agua se tomaron a media columna con una botella Van Dorn de $2.5 \mathrm{~L}$ y el sedimento con una draga Petite Ponar. La materia orgánica se cuantificó mediante el método propuesto por Walkley y Black (Sparks et al., 1996).

Captura de organismos. Los organismos de la infauna se recolectaron mediante un nucleador Ljkelkamp® de $5 \mathrm{~cm}$ de diámetro con un área de $0.0019 \mathrm{~m}^{2}$ y una draga Petite Ponar con un área de $0.025 \mathrm{~m}^{2}$; para la epifauna se empleó una red de cuchara de $0.5 \mathrm{~m}^{2}$ de área, una red de barra tipo

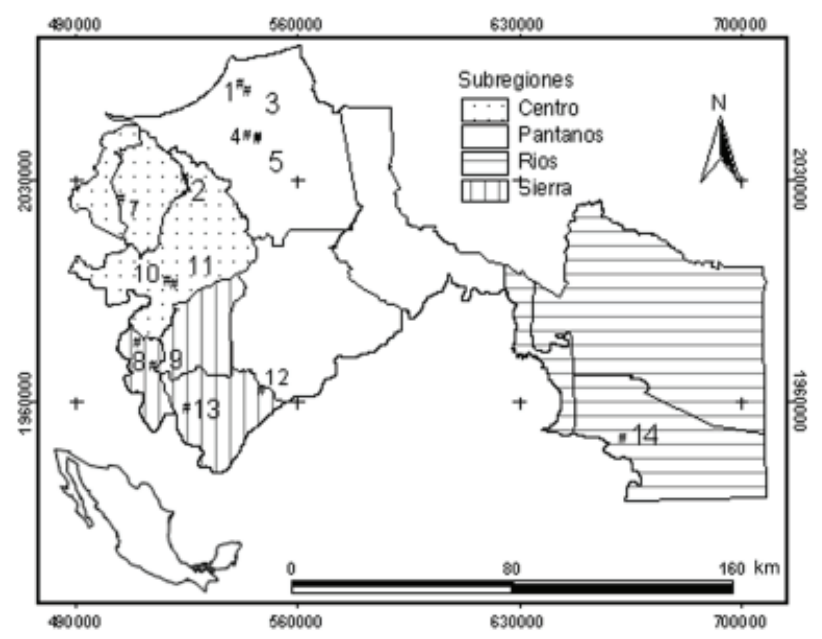

Figura 1. Localidades de muestreo en las subregiones del estado de Tabasco. 
Cuadro 1. Ubicación de las localidades de muestreo en los humedales de Tabasco por Municipio y subregión

\begin{tabular}{clcccc}
\hline & Localidad & Municipio & Subregión & \multicolumn{2}{c}{ UTM } \\
\hline 1 & Río Frontera (RF) & Centla & Pantanos & 536152 & 2046984 \\
2 & Simón Sarlat (SS) & Centla & Pantanos & 517914 & 2016820 \\
3 & Arroyo Polo (AP) & Centla & Pantanos & 536916 & 2046186 \\
4 & Canal (C) & Centla & Pantanos & 540905 & 2031441 \\
5 & La Puerta 3 (LP3) & Centla & Pantanos & 542256 & 2030860 \\
6 & Laguna San Pedrito (LSP) & Centla & Pantanos & 542197 & 2030983 \\
7 & Río Carrizal (RC) & Nacajuca & Centro & 496504 & 2010101 \\
8 & Río Teapa (RT) & Teapa & Sierra & 508893 & 1962375 \\
9 & Dren Artificial 1 (DA1) & Teapa & Sierra & 507159 & 1953770 \\
10 & R. Puyacatengo (RP) & Tacotalpa & Sierra & 511583 & 1945536 \\
11 & Dren Artificial 2 (DA2) & Tacotalpa & Sierra & 513440 & 1944922 \\
12 & Río Puxcatán (RPX) & Tacotalpa & Sierra & 544152 & 1944720 \\
13 & Arroyo Madrigal (AM) & Tacotalpa & Sierra & 518544 & 1939241 \\
14 & Arroyo Polevá (PVA) & Tenosique & Ríos & 666681 & 1928731 \\
\hline
\end{tabular}

Renfro con un área de barrido de $50 \mathrm{~m}^{2}$ (Renfro, 1962), así como recolectas manuales. Las muestras se fijaron con formalina al $4 \%$ para su posterior procesamiento. Los individuos fueron separados utilizando un tamiz con luz de malla de 0.5 y $0.75 \mu \mathrm{m}$, y preservados en alcohol al $70 \%$.

Los organismos se identificaron hasta el menor nivel taxonómico posible, mediante las claves de Bousfield (1972), Felder (1973), Pennak (1978), Williams (1984), Thorp y Covich (1991), Raz-Guzman et al. (1992), RazGuzman y Sánchez (1996) y Villalobos (1998). Para el arreglo sistemático se siguió lo propuesto por Martin y Davis (2001). Los organismos se encuentran depositados en la Colección de Referencia del Laboratorio de Ecología Acuática de ECOSUR- Unidad Villahermosa.

Análisis de datos. Las localidades de recolecta fueron agrupadas mediante un análisis de componentes principales (ACP), y para conocer la correlación existente entre las variables ambientales y la biota se aplicó un análisis de correspondencia canónica (ACC) que sirvió, a su vez, para determinar cuáles son las variables que tienen una mayor influencia en el ordenamiento de los patrones de datos de la comunidad (McGarigal et al., 2000). Los datos de las variables ambientales y la abundancia de los organismos fueron transformados a $\mathrm{Ln}(\mathrm{N}+1)$. La dominancia de las especies en frecuencia y abundancia se determinó mediante el ordenamiento de Olmstead-Tukey (Sokal y Rohlf, 1994), considerando especies dominantes, típicas, oportunistas y raras.

\section{Resultados}

Descripción ambiental. Las localidades de muestreo fueron caracterizadas de acuerdo con las condiciones físicoquímicas registradas. Se presentaron temperaturas cálidas de $23^{\circ} \mathrm{C}$ a $28^{\circ} \mathrm{C}$, con $\mathrm{pH}$ básico y una OD relativamente alta $(4.0$ - $6.0 \mathrm{mg} / \mathrm{l})$, con excepción de las localidades Canal (C) y río Teapa (RT), donde el OD fue menor (2.0 - $3.6 \mathrm{mg} / \mathrm{l})$. En la concentración de amonio se presentaron valores elevados $(2.04-2.85 \mathrm{mg} / \mathrm{l})$ en las localidades Canal (C), Puerta 3 (LP3), laguna San Pedrito (LSP), río Frontera (RF) y río Carrizal (RC). También los nitratos tuvieron valores elevados (1.51 - $6.25 \mathrm{mg} / \mathrm{l}$ ) (Cuadro 2).

La ordenación de las localidades mediante el ACP mostró que los 2 primeros componentes explicaron el $57.67 \%$ de la variación total; el primer componente conformó el $37.40 \%$ de acuerdo con las variables de amonio, nitratos y la profundidad, mientras que el segundo sumó el $20.27 \%$, representado por la temperatura y el pH (Cuadro 3). El primer grupo lo formaron las localidades 8 a 14, de las subregiones de Ríos (Tenosique) y Sierra (Teapa y Tacotalpa), y el segundo las localidades 1, 4, 5, 6 y 7 de la subregiónes Pantanos (Centla) y Centro (Villahermosa). La localidad 11 (dren artificial 2- DA2) quedó aislada al presentar un pH ácido de 5.87 (Fig. 2).

Composición y abundancia de crustáceos. Se recolectaron 2370 organismos correspondientes a 4 órdenes, 15 familias, 17 géneros y 17 especies; en la epifauna se contabilizaron 13 especies, mientras que 4 especies fueron para la infauna. Las especies mejor representadas en términos de abundancia relativa total fueron: Discapseudes holthuisi Bacescu y Gutu, 1975 (61.7\%), Macrobrachium acanthurus (Wiegmann, 1836) (11.7\%), Leptocheirus sp. Zaddach, 1844 (7.64\%) y Palaemonetes vulgaris (Say, 1818) (6.67\%), sumando el $87.71 \%$, el valor mínimo correspondió a Periclimenes americanus (Kingsley, 1878) 
Cuadro 2. Valores promedio de los parámetros físico-químicos en las localidades de muestreo y tipo de substrato (VFE= vegetación flotante emergente, $\mathrm{VC}=$ vegetación circundante, $\mathrm{VAS}$ = vegetación acuática sumergida y SSSV= substratos suaves sin vegetación)

\begin{tabular}{|c|c|c|c|c|c|c|c|c|c|}
\hline & $\begin{array}{l}\text { Localidad / variable } \\
\text { Unidades }\end{array}$ & $\begin{array}{c}\text { Temp. } \\
{ }^{\circ} \mathrm{C}\end{array}$ & $p H$ & $\begin{array}{l}O D \\
m g / l\end{array}$ & $\begin{array}{l}Z \\
m\end{array}$ & $\begin{array}{l}\text { Transp. } \\
\qquad m\end{array}$ & $\begin{array}{l}\mathrm{NH}_{4} \\
\mathrm{mg} / \mathrm{l}\end{array}$ & $\begin{array}{l}\mathrm{NO}_{3} \\
m g / l\end{array}$ & Substrato \\
\hline 1 & Río Frontera (RF) & 28.20 & 7.60 & 4.60 & 3.00 & 0.45 & 2.85 & 1.98 & VFE \\
\hline 2 & Simón Sarlat (SS) & 28.00 & 7.65 & 5.25 & 4.00 & 0.17 & 0.00 & 0.00 & VC, VFE \\
\hline 3 & Arroyo Polo (AP) & 27.00 & 7.66 & 5.50 & 2.00 & 0.85 & 0.00 & 0.00 & VAS \\
\hline 4 & Canal (C) & 28.50 & 7.58 & 3.60 & 3.00 & 0.75 & 2.27 & 1.70 & $\mathrm{VC}$ \\
\hline 5 & La Puerta 3 (LP3) & 26.20 & 7.87 & 5.04 & 2.10 & 0.95 & 2.04 & 1.51 & $\mathrm{VC}$ \\
\hline 6 & Laguna San Pedrito (LSP) & 27.00 & 7.58 & 6.00 & 1.50 & 0.75 & 4.28 & 5.50 & VAS \\
\hline 7 & Río Carrizal (RC) & 25.70 & 8.03 & 4.50 & 1.15 & 0.56 & 6.25 & 7.57 & SSSV \\
\hline 8 & Río Teapa (RT) & 25.50 & 7.66 & 2.00 & 1.50 & 0.45 & 0.00 & 0.00 & SSSV \\
\hline 9 & Dren Artificial 1 (DA1) & 25.70 & 7.76 & 4.00 & 1.50 & 0.85 & 0.00 & 0.00 & SSSV \\
\hline 10 & R. Puyacatengo (RP) & 26.93 & 7.54 & 4.00 & 1.00 & 0.55 & 0.00 & 0.00 & SSSV \\
\hline 11 & Dren Artificial 2 (DA2) & 28.16 & 5.87 & 4.00 & 1.55 & 0.75 & 0.00 & 0.00 & VAS \\
\hline 12 & Río Puxcatán (RPX) & 23.40 & 7.60 & 4.00 & 1.80 & 1.15 & 0.00 & 0.00 & SSSV \\
\hline 13 & Arroyo Madrigal (AM) & 24.00 & 8.00 & 4.00 & 1.30 & 0.70 & 0.00 & 0.00 & VAS \\
\hline 14 & Arroyo Polevá (APVA) & 27.00 & 7.15 & 4.00 & 1.10 & 1.00 & 0.00 & 0.00 & $\mathrm{VC}$ \\
\hline
\end{tabular}

Cuadro 3. Matriz factorial ambiental del ACP para las localidades de muestreo

\begin{tabular}{llr}
\hline & Eje 1 & Eje 2 \\
\hline Temp. & 0.219 & -0.623 \\
$\mathrm{pH}$ & 0.153 & 0.734 \\
$\mathrm{OD}$ & 0.300 & -0.041 \\
Profundidad & 0.351 & -0.190 \\
Transparencia & 0.195 & -0.109 \\
$\mathrm{NH}_{4}$ & 0.591 & 0.086 \\
$\mathrm{NO}_{3}$ & 0.572 & 0.128 \\
\hline
\end{tabular}

con $0.04 \%$ del total (Cuadro 4 ).

El análisis Olmstead-Tukey para la abundancia presentó 4 especies dominantes: M. acanthurus, Procambarus (Austrocambarus) llamasi Villalobos, 1954, D. holthuisi y Leptocheirus sp.; las oportunistas fueron 4: P. vulgaris, Hyalella azteca Zaddach, 1844, Leptochelia rapax (Harger, 1879) y Armases ricordi (H. Milne Edwards, 1853); hubo 2 especies típicas, Rhithropanopeus harrisii (Gould, 1841) y Potamocarcinus hartmanni Pretzmann, 1975 y 6 fueron raras (Fig. 3).

De acuerdo con sus hábitos, $D$. holthuisi fue especie dominante para la infauna con 1457 organismos; las oportunistas fueron Leptocheirus sp. y P. (Austrocambarus)

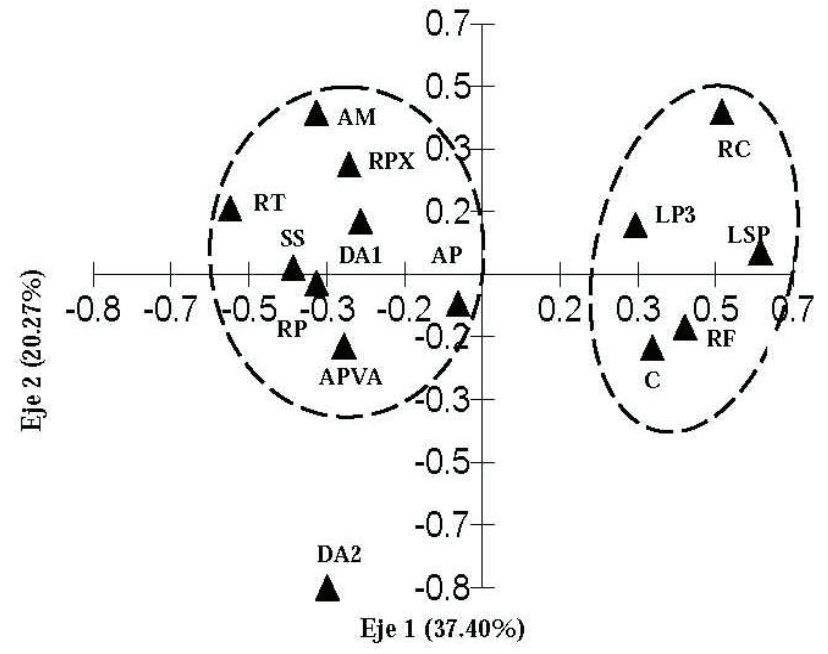

Figura 2. Ordenación de las localidades basada en las variables ambientales mediante el análisis de componentes principales (ACP).

llamasi, y 1 especie típica, L. rapax (Fig. 4). Para la epifauna se obtuvieron 2 especies dominantes, M. acanthurus y $R$. harrisi; 3 oportunistas, P. vulgaris, Leptocheirus sp. y A. ricordi; 3 típicas, P. hartmanni, Uca sp. Leach, 1814 y 
Potimirin mexicana (Saussure, 1857), y las especies raras fueron $H$. azteca, Processa sp. Leach, 1815 y P. hartmanni (Fig. 5).

Distribución de la abundancia por subregiones y ambientes. La distribución de los crustáceos en los humedales de Tabasco mostró que el $81 \%$ de la abundancia total se capturó en 4 localidades (C, LSP, LP3, LSP) de la subregión Pantanos en sistemas lénticos; la localidad con el máximo valor de abundancia correspondió a la LSP con 1534 individuos, mientras que el mínimo fue para LP3 con 11 individuos (Cuadro 5).

En relación con el número de especies, la localidad LSP (subregión Pantanos), presentó 6 especies: Callinectes similis (Williams, 1974) y $R$. harrisi registradas sólo para este sistema; D. holthuisi, Leptocheirus sp., M. acanthurus y Sphaeroma terebrans (Bate, 1866), especies compartidas con las localidades LSP y C. Para la localidad SS se capturaron de manera exclusiva $H$. azteca y Procambarus llamasi, y en LP3, las especies D. holthuisi y S. terebrans (Cuadro 5).

Los sistemas lóticos constaron de 10 localidades y contribuyeron con el $20 \%$ del total de organismos. Espacialmente, las localidades AP-3, RF y DA2, mostraron la mayor abundancia de organismos, con 296 (63\%), 61 (13\%) y 49 (10\%) de manera respectiva; mientras que las localidades RC y RT presentaron la menor abundancia con
1 organismo cada una. En cuanto al número de especies, el máximo correspondió a AP con 9, y en 6 localidades sólo se registró 1 especie (Cuadro 5).

Agrupaciones faunísticas. El ACP para infauna presentó 2 agrupaciones, la primera conformada por las localidades C, LSP y LP3 y la segunda por la localidad AP (subregión Pantanos). Los 2 primeros componentes conformaron el $92.57 \%$ de la variación total (Fig. 6). El ACC de la infauna y las variables ambientales se explica por los 2 primeros componentes con un $98.5 \%$ de la variación total, donde las variables amonio, nitratos, profundidad y temperatura determinaron esta ordenación (Cuadro 6). La especie $L$. rapax se correlacionó negativamente con estas variables y positivamente con el $\mathrm{pH}$, mientras que $D$. holthuisi se correlacionó con la temperatura. Leptocheirus sp. Se asoció positivamente con el OD y negativamente con la temperatura y la profundidad (Fig. 7).

El ACP aplicado a la epifauna, conformó 3 grupos que explicaron el $52.88 \%$ de la variación total en los 2 primeros componentes (Fig. 8). El primer grupo lo conformaron las localidades C, LP3, LSP (subregión Pantanos) en ambientes lénticos, con abundancias máximas; un segundo grupo, las localidades AP y RF en ambientes lóticos y con influencia salina. La última agrupación la constituyeron el resto de las localidades pertenecientes a las subregiones Sierra, Centro y Ríos en ambientes lóticos (Fig. 9).

Cuadro 4. Composición taxonómica, abundancia total y relativa de los crustáceos en los humedales de Tabasco

\begin{tabular}{|c|c|c|c|c|c|}
\hline Orden & Familia & Género & Especie & Abundancia & Abundancia \\
\hline 4 & 15 & 17 & 17 & núm. organismos & \\
\hline \multirow[t]{2}{*}{ Amphipoda } & Aoridae & Leptocheirus & sp. Zaddach, 1844 & 181 & 7.64 \\
\hline & $\begin{array}{l}\text { Gammaridae } \\
\text { Ischyroceridae }\end{array}$ & $\begin{array}{l}\text { Hyalella } \\
\text { Erichthonius }\end{array}$ & $\begin{array}{c}\text { azteca Saussure, } 1857 \\
\text { brasiliensis (Dana, 1852) }\end{array}$ & $\begin{array}{r}33 \\
2\end{array}$ & $\begin{array}{l}1.39 \\
0.08\end{array}$ \\
\hline Isopoda & Sphaeromatidae & Sphaeroma & terebrans Bate, 1866 & 8 & 0.34 \\
\hline \multirow[t]{2}{*}{ Tanaidacea } & Apseudidae & Discapseudes & holthuisi Bacescu y Gutu, 1975 & 1464 & 61.77 \\
\hline & Pseudozeuxidae & Leptochelia & rapax (Harger, 1879) & 62 & 2.62 \\
\hline \multirow[t]{11}{*}{ Decapoda } & Atyidae & Potimirim & mexicana (Saussure 1857) & 2 & 0.08 \\
\hline & Cambaridae & Procambarus & llamasi Villalobos, 1954 & 85 & 3.59 \\
\hline & Grapsidae & Armases & ricordi (H. Milne Edwards, 1853) & 38 & 1.60 \\
\hline & Palaemonidae & Macrobrachium & acanthurus (Wiegmann, 1836) & 278 & 11.73 \\
\hline & & Palemonetes & vulgaris (Say, 1818) & 158 & 6.67 \\
\hline & & Periclimens & americanus (Kingsley, 1878) & 1 & 0.04 \\
\hline & Portunidae & Callinectes & similis Williams, 1974 & 2 & 0.08 \\
\hline & Processidae & Processa & sp. Leach, 1815 & 12 & 0.51 \\
\hline & Pseudothelphusidae & Potamocarcinus & hartmanni (Pretzmann, 1975) & 14 & 0.59 \\
\hline & Xanthidae & Rhithropanopeus & harrisii (Gould, 1841) & 24 & 1.01 \\
\hline & Ocypodidae & $U c a$ & sp. Leach, 1814 & 6 & 0.25 \\
\hline
\end{tabular}




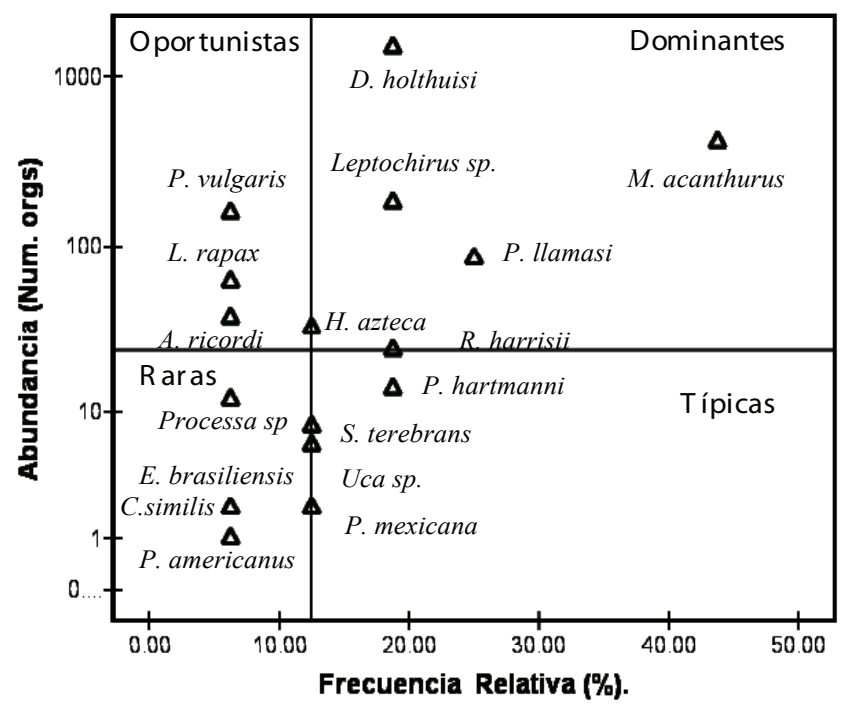

Figura 3. Jerarquización de las especies de crustáceos en los humedales del estado de Tabasco por su abundancia.

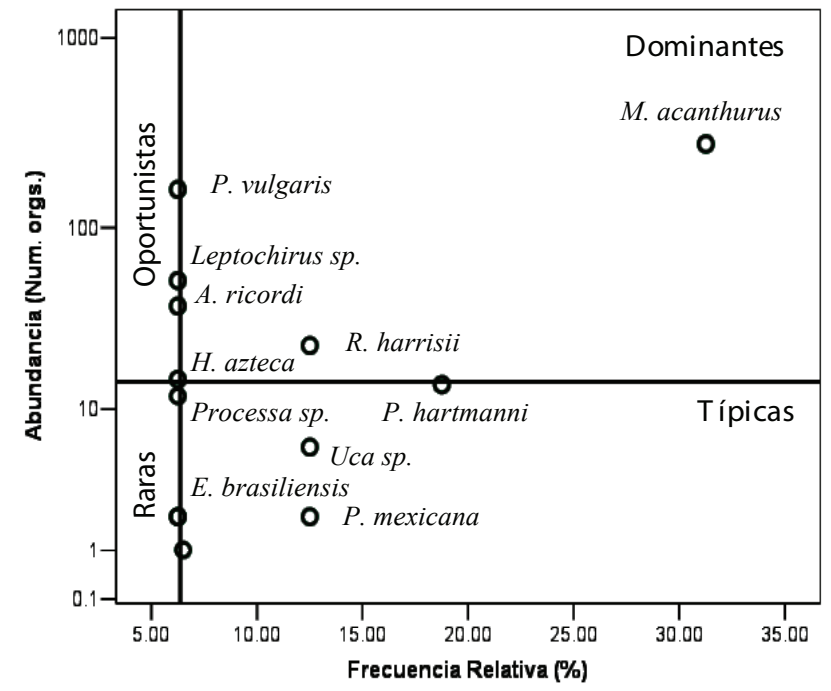

Figura 5. Jerarquización de las especies de crustáceos de la epifauna en los humedales del estado de Tabasco por su abundancia.

En la epifauna el ACC explicó el 52.88\% de la variación total en los 2 primeros componentes (Fig. 9). Los nutrientes OD y profundidad tuvieron mayor influencia en la ordenación y distribución de las especies (Cuadro 7). Las especies M. acanthurus, S. terebrans, P. llamasi y C. similis presentaron correlación positiva con los nitratos y amonio; $R$. harrisii y $U c a$ sp. se correlacionaron positivamente con el $\mathrm{pH}$ y el $\mathrm{OD}$; mientras que $P$. hartmanni y $P$. mexicana

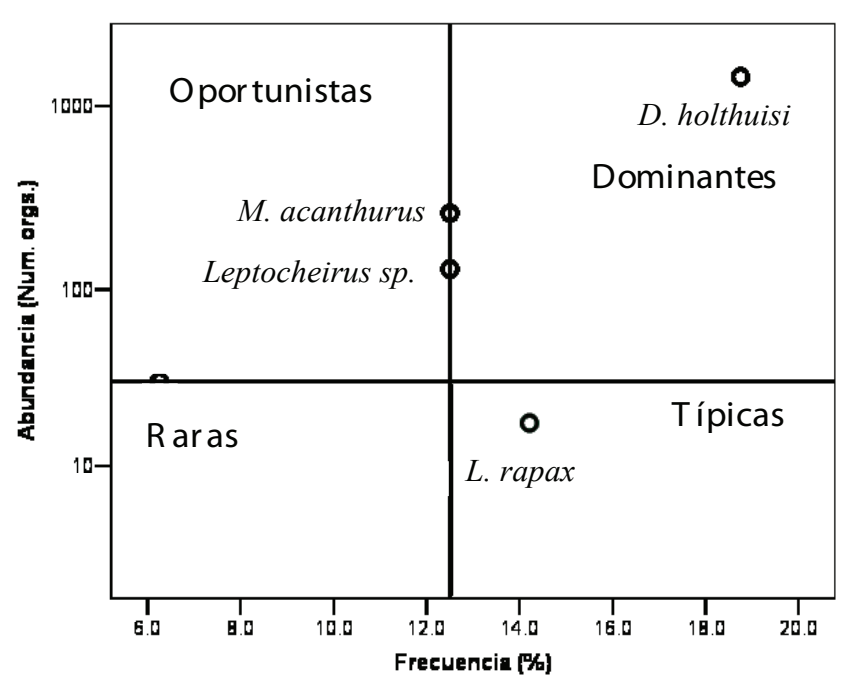

Figura 4. Jerarquización de las especies de crustáceos de la infauna en los humedales del estado de Tabasco por su abundancia.

Cuadro 5. Número de especies, abundancia y hábitos de los crustáceos por localidad y subregión. ( $\mathrm{R}=$ número de especies, $\mathrm{N}=$ número de organismos, Hábito: $\mathrm{E}=$ epifauna, $\mathrm{I}=$ infauna)

\begin{tabular}{ccccccc}
\hline Localidad & Municipio & Subregión & $R$ & $N$ & $\begin{array}{c}\text { Hábito } \\
\text { E/I }\end{array}$ \\
\hline & & & & & \\
2 & LS & Centla & Pantanos & 2 & 33 & $31 / 2$ \\
4 & C & Centla & Pantanos & 4 & 321 & $202 / 119$ \\
5 & LP3 & Centla & Pantanos & 1 & 11 & $0 / 11$ \\
6 & LSP & Centla & Pantanos & 6 & 1534 & $71 / 1463$ \\
& & & Lóticos & & & \\
1 & RF & Centla & Pantanos & 4 & 61 & $61 / 10$ \\
3 & AP & Centla & Pantanos & 9 & 296 & $182 / 114$ \\
7 & RC & Nacajuca & Centro & 1 & 1 & $1 / 0$ \\
8 & RT & Teapa & Sierra & 1 & 1 & $1 / 0$ \\
9 & DA1 & Teapa & Sierra & 2 & 18 & $1 / 17$ \\
10 & RP & Tacotalpa & Sierra & 3 & 20 & $3 / 17$ \\
11 & DA2 & Tacotalpa & Sierra & 1 & 49 & $0 / 49$ \\
12 & RPx & Tacotalpa & Sierra & 1 & 11 & $11 / 0$ \\
13 & AM & Tacotalpa & Sierra & 1 & 11 & $7 / 0$ \\
14 & APva & Tenosique & Ríos & 1 & 7 & $7 / 0$ \\
\hline & & & & &
\end{tabular}




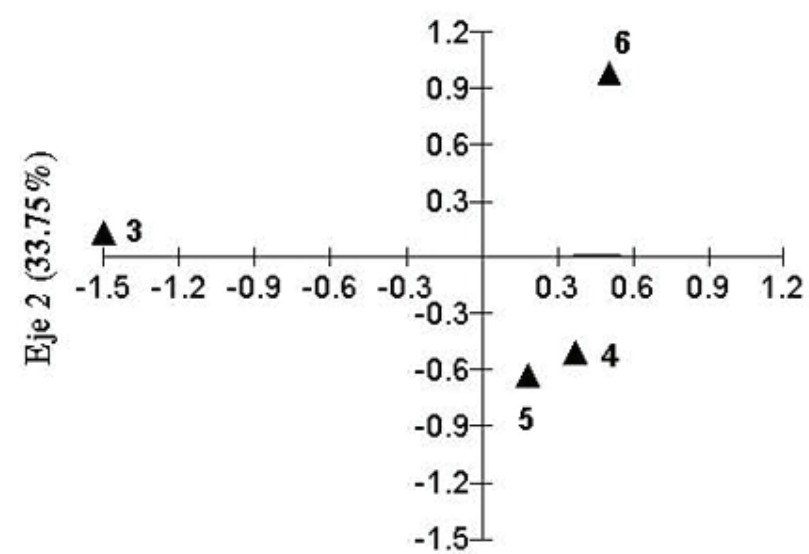

Eje $1(58.82 \%)$

Figura 6. Ordenación de las localidades basada en la abundancia de organismos de la infauna mediante el Análisis de Componentes Principales (ACP).

Cuadro 6. Coeficientes canónicos del ACC correspondiente a la infauna y variables ambientales de los dos primeros componentes

\begin{tabular}{lll}
\hline & Eje 1 & Eje 2 \\
\hline Temperatura & -0.424 & 0.360 \\
$\mathrm{pH}$ & 0.334 & 0.450 \\
$\mathrm{OD}$ & 0.419 & -0.795 \\
Profundidad & -0.234 & 0.864 \\
Transparencia & -0.495 & -0.472 \\
$\mathrm{NH}_{4}$ & -0.890 & -0.430 \\
$\mathrm{NO}_{3}$ & -0.787 & -0.615 \\
\hline
\end{tabular}

mostraron correlación negativa. Finalmente, H. azteca y $A$. ricordi tuvieron correlación positiva con la temperatura y negativa con los nutrientes (Fig. 9).

\section{Discusión}

Caracterización ambiental. En general las localidades presentaron buenas condiciones en cuanto a las concentraciones de oxígeno disuelto, con excepción de $\mathrm{C}$ y RT, que las tuvieron por debajo del mínimo requerido para la protección de la vida acuática. En contraste, el amonio y los nitratos sobrepasaron los límites máximos permisibles

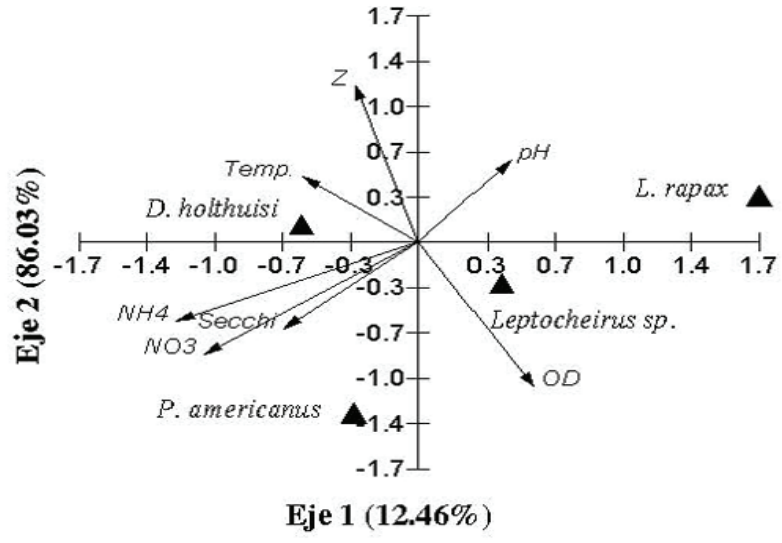

Figura 7. Ordenación de las especies de crustáceos basada en la matriz ambiental y biológica.

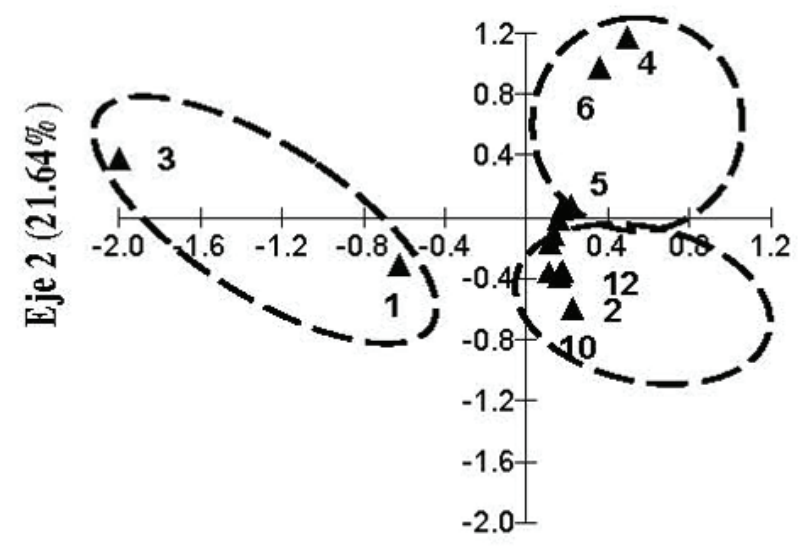

Eje $1(31.24 \%)$

Figura 8. Ordenación de las localidades basada en la abundancia de la infauna mediante el Análisis de Componentes Principales (ACP).

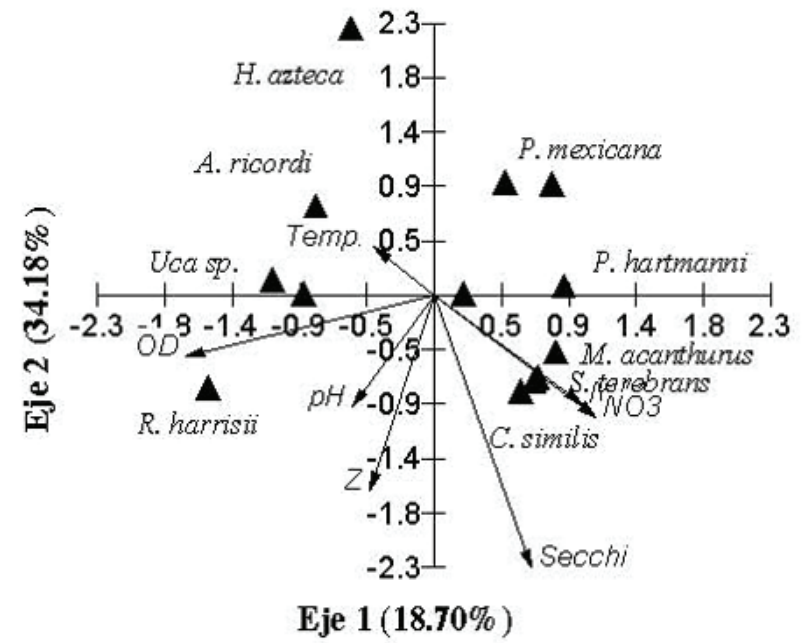

Figura 9. Ordenación de las localidades basada en la abundancia de la epifauna mediante el Análisis de Componentes Principales (ACP). 
para la protección de la vida acuática (SEDUE, 1989; CNA, 2003), en las localidades RF, SS, C, LP3, LSP (subregión Pantanos) y RC (subregión Centro). Esto podría explicarse debido a la posición geográfica de las localidades en la cuenca baja del sistema Grijalva-Usumacinta, donde se reciben los aportes provenientes de las cuencas alta y media. La zona alta y media presentan zonas con diferentes usos de suelos, donde destacan los cultivos y diferentes actividades antrópicas, así como descargas de la ciudad de Villahermosa. Aunque la Reserva de la Biosfera Pantanos de Centla es un área natural protegida, se realizan diferentes actividades dentro de ella, siendo las principales amenazas el cambio del flujo hidrológico y de uso de suelo para agricultura y ganadería (Hargrave, 2001).

La localidad DA2 se ubicó de manera aislada del grupo debido a su valor de $\mathrm{pH}$ ácido, lo que pudiera relacionarse con la descomposición de la vegetación acuática sumergida y liberación de taninos en esta localidad. Dicho ordenamiento fue confirmado mediante el ACP, separó a las localidades en 2 grupos asociando las localidades RF, C, LP3 y LSP (subregión Pantanos), situadas en áreas asociadas con asentamientos humanos y/o parcelas de cultivo. Estas actividades generan un cambio en el uso de suelo, en especial en las márgenes ribereñas, donde la pérdida de cobertura vegetal disminuye la cantidad de hábitats acuáticos disponibles, incrementan la tasa de sedimentación y provocan valores bajos en la abundancia y riqueza de macroinvertebrados (Sharitz y Batzer, 1999) y peces dulceacuícolas, que deterioran la estructura trófica (Barnes y Mann, 2001).

Composición faunística. El mayor número de especies de la epifauna se puede explicar por los flujos hidrológicos de la región, así como por la disponibilidad de sustratos con vegetación y microhábitats, que proveen áreas de reproducción y protección para un elevado número de organismos (Williams, 1980; MacFarlane, 1983; Bailey y Litterick, 1993, Covich et al., 1999). El menor número de especies de la infauna puede deberse a los aportes

Cuadro 7. Coeficientes canónicos del ACC correspondiente a la epifauna y variables ambientales de los dos primeros componentes

\begin{tabular}{lcc}
\hline & Eje 1 & Eje 2 \\
\hline Temperatura & -0.106 & -0.314 \\
$\mathrm{pH}$ & -0.058 & -0.461 \\
$\mathrm{OD}$ & -0.951 & -0.195 \\
Profundidad & -0.646 & -0.969 \\
Transparencia & 0.289 & -0.426 \\
$\mathrm{NH}_{4}$ & -0.776 & 5.218 \\
$\mathrm{NO}_{3}$ & 1.559 & -4.949 \\
\hline
\end{tabular}

de sedimentos y material en suspensión proveniente de cuenca arriba y con ello gran cantidad de agroquímicos, y a la homogeneidad del sustrato (Malavoi y Souchon, 2002) y consumo por depredadores (Barba et al., 2000). La comparación del número de especies por subregión mostró qué pantanos tuvieron los valores máximos debido a una mayor disponibilidad de hábitat dada por la vegetación de manglar, vegetación emergente, flotante y vegetación acuática sumergida, en las que se asocia una mayor diversidad y abundancia de organismos (Macinthosh et al., 1999; Barba, 2005; Barba y Sánchez, 2005; Barba et al., 2005), así como al transporte pasivo mediante la vegetación acuática flotante (Tankersley et al., 2002).

Distribución y ecología. Presentaron distribución restringida a ambientes tropicales y dulceacuícolas 3 especies: Procambarus llamasi, dulceacuícola de zonas inundadas y pantanos (Hobbs, 1989); P. mexicana, de arroyos de montaña hasta grandes ríos cercanos a la costa (Martínez-Mayén y Román-Contreras, 2005) y $P$. hartmanni, de aguas someras y rocosas, en aguas limpias con restos de vegetación (Álvarez et al., 2005).

Las especies Erichtonius brasiliensis (Dana, 1852), D. holthuisi, M. acanthurus, P. vulgaris, P. americanus, $R$. harrisi, L. rapax, S. terebrans, C. similis, A. ricordi, Uca sp., Processa sp. y Leptocheirus sp., son especies con una amplia distribución y tolerancia a ambientes oligohalinos y costeros, asociados a sustratos con vegetación marginal y circundante, así como sumergida y flotante (Sánchez et al., 1998; Escobar-Briones et al., 1999).

De acuerdo con Álvarez et al. (2005), están registradas 72 especies de crustáceos, de los cuáles 44 contabilizaron para decápodos y peracáridos. En este trabajo se listan 17 especies para ambos grupos (Cuadro 8); pese a que se lista un menor número, se agregan al inventario las especies $E$. brasiliensis, Leptocheirus sp., P. vulgaris, P. americanus y Processa sp. Se considera que existe una subestimación de los registros para invertebrados y en especial de crustáceos, ya que la mayoría de los estudios se han efectuado con especies de importancia comercial (Sánchez y Barba, 2005).

Agrupaciones faunísticas. De acuerdo con el ACP, se conformaron 3 grupos; el primero con las localidades C, LP3 y LSP, pertenecientes a las subregiones Pantanos y Centro; el segundo con las localidades de las subregiones Centro y Ríos. La separación de las localidades AP y RF (subregión Pantanos) se debió a registros únicos para estos sitios, representados para RF por las especies $U c a$ sp. y Processa sp., y para AP (subregión Pantanos) por $L$. rapax, $P$. vulgaris y $P$. americanus, que combinados con la influencia salina de la boca del río Frontera marcaron una diferencia.

Las agrupaciones obtenidas por el ACC mostraron 
que los nutrientes fueron los factores determinantes para su formación, donde D. holthuisi, M. acanthurus,
S. terebrans y C. simillis, caracterizaron la subregión de Pantanos, siendo estos sitios los de mayores valores en

Cuadro 8. Registro de crustáceos para Tabasco (1= Álvarez et al., 2005; 2= este estudio)

\begin{tabular}{|c|c|c|}
\hline Crustáceos Peracáridos y Decápodos & 1 & 2 \\
\hline Hyalella azteca Saussure, 1857 & $\mathrm{X}$ & $\mathrm{X}$ \\
\hline Platorchestia platensis (Kroyer, 1845) & $\mathrm{X}$ & \\
\hline Erichthonius brasiliensis (Dana, 1853) & & $\mathrm{X}$ \\
\hline Leptocheirus sp Zaddach, 1844 & & $\mathrm{X}$ \\
\hline Sphaeroma terebrans Bate, 1866 & $\mathrm{X}$ & $\mathrm{X}$ \\
\hline Discapseudes holthuisi Bacescu y Gutu, 1975 & $\mathrm{X}$ & $\mathrm{X}$ \\
\hline Leptochelia rapax Harger, 1879 & & $\mathrm{X}$ \\
\hline Farfantepenaeus aztecus (Ives, 1891) & $\mathrm{X}$ & \\
\hline Farfantepenaeus duorarum (Burkenroad, 1939) & $\mathrm{X}$ & \\
\hline Litopenaeus setiferus (Linnaeus, 1767) & $\mathrm{X}$ & \\
\hline Xiphopenaeus kroyeri (Heller, 1862) & $X$ & \\
\hline Lucifer faxoni Borradaile, 1915 & $\mathrm{X}$ & \\
\hline Atya scabra Leach, 1815 & $\mathrm{X}$ & \\
\hline Potimirim mexicana (Saussure, 1857) & $\mathrm{X}$ & $\mathrm{X}$ \\
\hline Macrobrachium acanthurus (Wiegmann, 1836) & $\mathrm{X}$ & $\mathrm{X}$ \\
\hline Macrobrachium acherontium Holthuis, 1977 & $\mathrm{X}$ & \\
\hline Macrobrachium carcinus (Linnaeus, 1758) & $\mathrm{X}$ & \\
\hline Macrobrachium heterochirus (Wiegmann, 1836) & $\mathrm{X}$ & \\
\hline Macrobrachium hobbsi Nates y Villalobos, 1990 & $\mathrm{X}$ & \\
\hline Macrobrachium olfersii (Wiegmann, 1836) & $\mathrm{X}$ & \\
\hline Palaemonetes vulgaris (Say, 1818) & & $\mathrm{X}$ \\
\hline Periclimenes americanus (Kingsley, 1878) & & $\mathrm{X}$ \\
\hline Processa sp. Leach, 1815 & & $\mathrm{X}$ \\
\hline Procambarus (Austrocambarus) acanthophorus Villalobos, 1948 & $\mathrm{X}$ & \\
\hline Procambarus (Austrocambarus) llamasi Villalobos, 1954 & $\mathrm{X}$ & $\mathrm{X}$ \\
\hline Callinectes rathbunae Contreras, 1930 & $\mathrm{X}$ & \\
\hline Callinectes sapidus Rathbun, 1895 & $\mathrm{X}$ & \\
\hline Callinectes similis Williams, 1974 & $\mathrm{X}$ & $\mathrm{X}$ \\
\hline Rhithropanopeus harrisii (Gould, 1841) & $\mathrm{X}$ & $\mathrm{X}$ \\
\hline Cardisoma guanhumi Latreille, 1825 & $\mathrm{X}$ & \\
\hline Platychirograpsus spectabilis de Man, 1896 & $\mathrm{X}$ & \\
\hline Aratus pisonii (H. Milne Edwards, 1837) & $\mathrm{X}$ & \\
\hline Armases americanum (Saussure, 1858) & $\mathrm{X}$ & \\
\hline Armases cinereum (Bosc, 1802) & $\mathrm{X}$ & \\
\hline Armases ricordi (H. Milne Edwards, 1853) & $X$ & $\mathrm{X}$ \\
\hline Goniopsis cruentata (Latreille, 1802) & $\mathrm{X}$ & \\
\hline Sesarma crassipes Cano, 1889 & $\mathrm{X}$ & \\
\hline Sesarma curacaoense de Man, 1892 & $\mathrm{X}$ & \\
\hline Uca burgersi Holthuis, 1967 & $\mathrm{X}$ & \\
\hline Uca marguerita Thurman, 1981 & $\mathrm{X}$ & \\
\hline Uca pugnax (Smith, 1870) & $\mathrm{X}$ & \\
\hline Uca rapax (Smith, 1870) & $\mathrm{X}$ & \\
\hline Uca vocator (Herbst, 1804) & $\mathrm{X}$ & \\
\hline Uca sp. Leach, 1814 & & $\mathrm{X}$ \\
\hline Ucides cordatus (Linnaeus, 1763) & $\mathrm{X}$ & \\
\hline Potamocarcinus hartmanni Pretzmann, 1975 & $\mathrm{X}$ & $\mathrm{X}$ \\
\hline Odontothelphusa maxillipes (Rathbun, 1898) & $\mathrm{X}$ & \\
\hline Odontothelphusa monodontis Rodríguez y Hobbs, 1989 & $\mathrm{X}$ & \\
\hline Tehuana chontalpaensis Villalobos y Álvarez, 2003 & $\mathrm{X}$ & \\
\hline Avotrichodactylus bidens (Bott, 1969) & $\mathrm{X}$ & \\
\hline \multirow[t]{2}{*}{ Avotrichodactylus constrictus (Pearse, 1911) } & $\mathrm{X}$ & \\
\hline & Número total & 17 \\
\hline
\end{tabular}


estas variables; Uca sp. y R. harrisi se correlacionaron con el OD y Armases ricordi con la temperatura. Sin embargo, los resultados aquí obtenidos mostraron que las variables de mayor importancia fueron los nutrientes, y en menor proporción la temperatura y el OD (Williams, 1980; Statzner et al., 1988; Allan, 1995).

Lo anterior sugiere la necesidad de ampliar el estudio de otros humedales debido a la importancia de estos hábitats en cuanto a su cobertura en Tabasco. Por otro lado, el conocimiento de los crustáceos y otros invertebrados acuáticos como organismos bioindicadores en los humedales constituye una herramienta potencial para caracterizar el estado de salud de estos ambientes.

\section{Agradecimientos}

Los autores agradecemos los apoyos otorgados por los proyectos: "Programa de consolidación institucional: investigadores mexicanos del CONACYT". Convenio específico Ref. 323201-1 Exp. 020314; “Taller sobre manejo de bordos en el estado de Tabasco" (SEDAFOPECOSUR); "Conservación y mejoramiento de suelos en Tabasco: tecnologías para el restablecimiento a la fertilidad de suelos y para controlar la erosión hídrica" (SEDAFOP-ECOSUR), e "Inventario de las microcuencas del municipio de Balancán, Tabasco" (Fondo MixtoCONACYT -Gobierno del Estado de Tabasco TAB2006-C06-46385). Por otro lado, las facilidades otorgadas al Posgrado de ECOSUR para la realización de salidas a campo del curso Humedales: ecología y manejo, de la Maestría en Ciencias Naturales y Desarrollo Rural.

\section{Literatura citada}

Allan, J. D. 1995. Stream ecology, structure and function. Chapman 6 Hall, London. $350 \mathrm{p}$.

Álvarez, F., J. L. Villalobos y E. Lira. 1996. Decapoda. In Biodiversidad, taxonomía y biogeografía de artrópodos de México: hacia una síntesis de su conocimiento, vol. I, J. E. Llorente, A. N. García-Aldrete y E. González (eds.). Comisión Nacional para el Conocimiento y Uso de la Biodiversidad / Universidad Nacional Autónoma de México, México, D.F. p. 103-129.

Álvarez, F., J. L. Villalobos, y R. Robles. 2005. Crustáceos. In Biodiversidad del estado de Tabasco, Cap. 8, J. Bueno, F. Álvarez y S. Santiago (eds.). Instituto de Biología,UNAM/ Comisión Nacional para el Conocimiento y Uso de la Biodiversidad, México, D.F. p. 177-194.

Araúz, B., R. Amores y E. Medianero. 2000. Diversidad y distribución de insectos acuáticos a lo largo del cauce del río Chico (Provincia de Chiriquí, República de Panamá). Scientia 15:27-46.
Bailey, R. G. y M. R. Litterick. 1993. The macroinvertebrate fauna of water hyacinth fringes in the Sudd swamps (Nile River, Southern Sudan). Hydrobiologia 250:97-103.

Barba, E. 2005. Valor del hábitat: Distribución de peces en humedales de Tabasco. ECOfronteras 25:9-11.

Barba, E. y A. J. Sánchez. 2005. Peracarid crustaceans of central Laguna Madre, Tamaulipas, in the southwestern Gulf of Mexico. Gulf of Mexico Science 23:241-247.

Barba, E., A. Raz-Guzman y A. J. Sánchez. 2005. Distribution patterns of estuarine caridean shrimps in the southwestern Gulf of Mexico. Crustaceana 78:709-726.

Barba, E., J. Rangel-Mendoza y R. Ramos-Reyes. 2006. Clasificación de los humedales de Tabasco mediante sistemas de información geográfica. Universidad y Ciencia 22:101-110.

Barba, E., A. J. Sánchez, A. Raz-Guzman y M. E. Gallegos. 2000. Dieta natural y tasa de forrajeo del carideo Hippolyte zostericola Smith sobre epífitas de Thalassia testudinum Banks et Solander ex König. Hidrobiológica 10:139-146.

Barnes, R. S. K. y K. H. Mann. 2001. Fundamentals of aquatic ecology, segunda edición, Blackwell, Toronto, Ontario. $270 \mathrm{p}$.

Bousfield, E. L. 1972. Shallow-water, Gammaridean Amphipoda of New England. Comstock/Cornell University Press, Ithaca, New York 312 p.

Comisión Nacional del Agua. 2003. Ley federal de derechos y normas aplicables en materia de aguas nacionales y sus bienes públicos inherentes. Diario Oficial de la Federación, México,D.F., 2 de enero: 173-191.

Covich, A. P., M. A. Palmer y T. A. Crowl. 1999. The role of benthic invertebrate species in freshwater ecosystems. Bioscience 49:119-127.

de la Lanza, G. y J. L. García-Calderón. 1995. Lagos y presas de México. Centro de Ecología y Desarrollo, México, D.F. $320 \mathrm{p}$.

de la Lanza, G., S. H. Pulido y J. L. P. Carvajal. 2000. Organismos indicadores de la calidad del agua y de la contaminación (bioindicadores). Plaza y Valdez / Comisión Nacional del Agua, SEMARNAP/Instituto de Biología, UNAM, México, D.F. 633 p.

Díaz, H. y G. Rodríguez. 1977. The branchial chamber of some terrestrial and semiterrestrial crabs. Biological Bulletin 153:485-504.

Escobar-Briones, E., F. Álvarez y G. Salgado. 1999. Discapseudes holthisi (Crustacea: Tanaidacea) as an intermediate host of Caballerohynchus lamothei (Acanthocephala: Cavasomidae). Journal of Parasitology 85:134-137.

Felder, D. L. 1973. An annotated key to crabs and lobsters (Decapoda, Reptantia) from coastal waters of the northwestern Gulf of Mexico. Center for Wetland Resources, Lousiana State University, Baton Rouge. 103 p.

Hargrave, B. T. 2001. Impacts of man's activities on aquatic systems. In Fundamentals of aquatic ecology, R. S. K. Barnes y K. H. Mann (eds.). Blackwell, Toronto, Ontario. p. 245-264.

Hobbs, H. H., Jr. 1989. An illustrated checklist of the American crayfishes (Decapoda: Astacidae, Cambaridae, and Parastacidae). Smithsonian Contributions to Zoology 
480:1-236.

Hurtado, S., F. García-Trejo y P. J. Gutiérrez-Yurrita. 2005. Importancia ecológica de los macroinvertebrados bentónicos de la subcuenca del río San Juan, Querétaro, México. Folia Entomológica Mexicana 44:271- 286.

Instituto Nacional de Estadística y Geografía. 2008. http:// mapserver.inegi.gob.mx/geografia/espanol/datosgeogra/ basicos/estados/tab_geo.cfm; última consulta: 12.III.2009.

Macintosh, D., J. Goncalves, F. Soares, S. M. Moser y N. Paphavisit. 1999. Transport mechanisms of crab megalopae in mangrove ecosystems, with special reference to a mangrove estuary in Ranong, Thailand. In Mud crab aquaculture and biology, C. P. Keenan y A. Blackshaw (eds.). Proceedings of an International Scientific Forum held in Darwin, Australia, 21- 24 April 1997, ACIAR Proceedings, p. 178-186.

Malavoi, J. R. e Y. Souchon. 2002. Description standarisee des principaux faciès dècoulement observables en rivière: clè de dètermination qualitative et mesures physiques. Bulletin Francais de Peche et Pisciculture 365/366:357-372.

Mantel, L. H. y L. L. Farmer. 1983. Osmotic and ionic regulation. In The Biology of Crustacea, vol. 5, Internal anatomy and physiological regulation, D. Bliss y L. Mantel (eds.). Academic, New York. p. 53-161.

Martin, J. W. y G. E. Davis. 2001. An updated classification of the recent Crustacea. Natural History Museum of Los Angeles County, Science Series 39, 124 p.

Martínez-Mayén, M. y R. Román-Contreras. 2005. Notes on the fertility of Potimirim mexicana (De Saussure, 1857) (Decapoda, Atyidae) in Laguna de Términos, Gulf of Mexico. Crustaceana 78:29-40.

McFarlane, M. B. 1983. Structure of benthic macroinvertebrate communities in a midwestern plain stream. Freshwater Invertebrate Biology 2:147-153.

McGarigal, K., S. Cushman y S. Stanfford. 2000. Multivariate statistics for wildlife and ecology research. Springer, New York. 283 p.

Pennak, R. W. 1978. Fresh water invertebrates of the United States. Wiley, New York. 628 p.

Ramírez, J. y G. Roldán. 1989. Contribución al conocimiento limnológico y de los macroinvertebrados acuáticos de algunos ríos de la región del Uraba antioqueño. Actualidades Biológicas 18:113-121.

Raz-Guzman, A. y A. J. Sánchez. 1996. Catálogo ilustrado de cangrejos braquiuros (Crustacea) de la laguna de Tamiahua, Veracruz, México. Cuaderno 31. Instituto de Biología, Universidad Nacional Autónoma de México, 52 p.

Raz-Guzman, A., A. J. Sánchez y L. A. Soto.1992. Catálogo ilustrado de cangrejos braquiuros y anomuros (Crustacea) de la laguna de Alvarado, Veracruz, México. Cuaderno 14, Instituto de Biología, Universidad Nacional Autónoma de México, $51 \mathrm{p}$.

Renfro, W. C. 1962. Small beam net for sampling postlarval shrimp. Galveston Biological Lab. June 30, 1962. U. S. Fisheries and Wildlife Services Circular 161:86-67.

Rincón, M. E. 2003. Comunidad de insectos acuáticos de la quebrada Mamarramos (Boyacá, Colombia). Revista Colombiana de Entomología 14:3-13.

Sánchez, A. J. y E. Barba. 2005. Biodiversidad de Tabasco. In Biodiversidad del estado de Tabasco, J. Bueno, F. Álvarez y S. Santiago (eds.). Instituto de Biología, UNAM / CONABIO, México, D.F. p. 1-16.

Sharitz, R. R. y D. P. Batzer. 1999. An introduction to freshwater wetlands in North America and their invertebrates. In Invertebrates in freshwater wetlands of North America: ecology and management, D.P. Batzer, R.B. Rader y S.A. Wissinger (eds.). Willey, New York. p. 1-21.

Secretaría de Desarrollo Urbano y Ecología. 1989. Criterios Ecológicos de la Calidad del Agua. CE-CCA-001/89. Diario Oficial de la Federación, 13 diciembre de 1989.

Sparks D.L., A. L. Page, P. A. Helmke, R. H. Loeppert, P. N. Soltanpour, M. A. Tabatabai, C. T. Johnston y M. E. Sumner (eds.). 1996. Methods of soil analysis, part 3. Chemical methods. Soil Science Society of America Book Series, Madison, Wisconsin. $1390 \mathrm{p}$.

Sokal, R. R. y F. J. Rohlf. 1994. Biometry. The principles and practice of statistics in biological research, third edition. Freeman, New York 880 p.

Statzner, B., J. A. Gore y V. H. Resh. 1988. Hydraulic stream ecology: observed patterns and potential applications. Journal of the North American Benthological Society 7:307-360.

Tankersley, R. A., J. M. Welch y R. B. Forward. 2002. Settlement times of blue crab (Callinectes sapidus) megalopae during flood-tide transport. Marine Biology 141:863-875.

Thorp, J. H. y A. P. Covich. 1991. Ecology and classification of North American freshwater invertebrates. Academic, San Diego, California. 911 p.

Villalobos, J. L. 1998. Taxonomía de los crustáceos con énfasis en el orden Decapoda. Instituto de Biología, UNAM, México, D.F. $33 \mathrm{p}$

Williams, D. D. 1980. Some relationships between stream benthos and substrate heterogeneity. Limnology and Oceanography 25:166-172.

Williams, A. B. 1984. Shrimps, lobsters and crabs of the Atlantic coast of the Eastern United States, Maine to Florida. Smithsonian Institution, Washington, D.C. 550 p.

Yáñez-Arancibia, A. 1986. Ecología de la zona costera. Análisis de siete tópicos. A.G.T., México, D.F. 189 p. 